\title{
Supersymmetric minisuperspace with non-vanishing fermion number
}

\author{
András Csordás* and Robert Graham \\ Fachbereich Physik, Universität-Gesamthochschule Essen \\ 45117 Essen \\ Germany
}

\begin{abstract}
The Lagrangean of $N=1$ supergravity is dimensionally reduced to one (time-like) dimension assuming spatial homogeneity of any Bianchi type within class A of the classification of Ellis and McCallum. The algebra of the supersymmetry generators, the Lorentz generators, the diffeomorphism generators and the Hamiltonian generator is determined and found to close. In contrast to earlier work, infinitely many physical states with non-vanishing even fermion number are found to exist in these models, indicating that minisuperspace models in supergravity may be just as useful as in pure gravity.
\end{abstract}

Minisuperspace models have long served as an enormeously useful testing ground for new ideas in quantum gravity, ranging from explorations of its mathematical structure to investigation in the many open problems of quantum cosmology (see e.g. [1] 3]). More recently, also the quantum theory of supersymmetric minisuperspace models has attracted the interest of many authors (see e.g. [4- 16) for similar reasons. Our own interest was first aroused by the chaotic classical nature of the Bianchi type IX models and the discovery [7] that supersymmetric versions have simple explicit analytical solutions in the empty and filled fermion sectors which can be interpreted as wormhole states [9] and, in other cases, as Hartle-Hawking no-boundary states [16. However, it has been shown meanwhile by a simple scaling argument, that these special solutions found in the minisuperspace models have no direct counterpart in 4-dimensional supergravity because there states in the empty and filled sectors cannot exist [17] (for a dissenting opinion see 18 ). This rather recent result makes appear disturbing another result found some time ago \& and claimed as confirmed by several groups of authors [9] 12]: namely that the solutions in the empty and filled fermion sectors are the only solutions (i. e. the only physical states satisfying all constraints) for all supersymmetric minisuperspace models of Bianchi type in class A 19] (without matter-coupling and with the exception, with a certain operator ordering [11,12, of Bianchi type I which is, also for other reasons, very special in this class). An even stronger result of this type was reported

\footnotetext{
*Permanent address: Research Institute for Solid State Physics, P.O. Box 49, H1525 Budapest, Hungary
}

for anisotropic supersymmetric minisuperspace models with a non-vanishing cosmological constant [13 15]: the present consensus in the literature is that no physical states exist, in this case, at all. Taken together these results force the conclusion that the physical states found in the minisuperspace models have no counterpart in the full theory, and vice-versa, which would render supersymmetric minisuperspace models useless as models of full supergravity contrary to the situation in pure gravity.

However, doubts may be raised. The quoted results for the minisuperspace models are paradoxical from a general point of view: In comparison to pure gravity, so it would appear from these findings, these supersymmetric models are overconstrained for physically not yet well understood reasons, even though supergravity certainly has more physical degrees of freedom than gravity, not less, namely the two additional physical degrees of freedom per space-point of the Rarita-Schwinger field.

The present work has the purpose to clarify these issues for the supersymmetric models of Bianchi type within class A of the classification of Ellis and McCallum [19]. Here we shall restrict ourselves to the case of a vanishing cosmological constant. We have derived explicitely, in the metric representation, the dimensionally reduced generators of supersymmetry transformations, Lorentz transformations, and coordinate transformations within the homogeneity group; we have then determined their closed graded algebra explicitely. The form of this graded algebra allows us to determine the form of the nontrivial physical states in all sectors with even fermion number, which may take the values $0,2,4,6$ in these models. In all of these sectors nontrivial solutions are found to exist, of which infinitely many have fermion number 2 and 4. Comparing with the earlier work, where only states for fermion number 0 and 6 were found, we notice that there the Lorentz-invariant ansatz for solutions in the two- und four-fermion sector was too special, allowing in each sector for 2 Lorentz invariant amplitudes only, rather than the 15 permitted ones. In our solutions all the 15 Lorentz-invariant amplitudes of the two-fermion sector appear (similarly for the four-fermion sector) and are reexpressed in terms of a single amplitude which must satisfy a Wheeler-DeWitt equation. Therefore, the new type of solution we find to exist in the two- and fourfermion sectors permits the free choice of the fermion sector, corresponding to the choice of the initial state of the Rarita-Schwinger field, and, in addition, just as 
much freedom in the choice of initial conditions as the Wheeler-DeWitt equation of the corresponding Bianchi models in pure gravity. Moreover, the new physical states we find in the minisuperspace models are direct analogues of physical states in full supergravity. As a consequence, supersymmetric minisuperspace models recover their significance as models of full supergravity.

We now proceed to present some more detail, however, omitting technical points as much as possible. These we shall present in a full report of this work elsewhere. We shall base our work on the Lagrangean of $N=1 \mathrm{su}-$ pergravity given in 20 adopting all spinor conventions given there. The excellent account of the Hamiltonian form of $N=1$ supergravity in the metric representation given in 21,50 is also freely used in the following. In the metric representation the independent variables are taken to be the tetrad components $e_{p}{ }^{a}$ (with Einstein indices $p=1,2,3$ from the middle of the alphabet and Lorentz indices $a=0,1,2,3$ from the beginning of the alphabet) and the Grassmannian components $\psi_{p}{ }^{\alpha}$ (with spinor index $\alpha=1,2)$ of the Rarita-Schwinger field. The $e_{p}{ }^{a}$ form the metric tensor $h_{p q}=e_{p}{ }^{a} e_{q a}$ on the space-like homogeneity 3 -surfaces in the symmetric basis of 1-forms $\boldsymbol{\omega}^{p}$, satisfying $d \boldsymbol{\omega}^{p}=\frac{1}{2}\left(m^{p q} / h^{1 / 2}\right) \varepsilon_{q r s} \boldsymbol{\omega}^{r} \otimes \boldsymbol{\omega}^{s}$ in Bianchi type models of class A. Here $h=\operatorname{det} h_{p q}$ and $\epsilon_{q r s}$ denotes the components of the Levi-Civita tensor (not the tensor density). The constant symmetric matrix $m^{p q}$ is fixed by the chosen Bianchi type [22]. It transforms as a tensor under all coordinate changes from one symmetric basis to another one. Due to the choice of a symmetric basis the $e_{p}{ }^{a}$ and $\psi_{p}{ }^{\alpha}$ are functions of time only.

Starting from the supergravity Lagrangean in [20], a canonical formulation of supergravity, restricted to the purely time-dependent variables $e_{p}{ }^{a}, \psi_{p}{ }^{\alpha}$, can now be developed in the same way as in [21]. From the Lagrangean one defines, as usual, the generalized momenta $\hat{p}^{p}{ }_{a}$ and $\hat{\pi}^{p}{ }_{\alpha}$ of $e_{p}{ }^{a}$ and $\psi_{p}{ }^{\alpha}$, respectively. The Poisson brackets must be replaced by Dirac brackets due to the appearance and subsequent elimination of second class constraints, by which the adjoint quantities $\bar{\psi}_{p}{ }^{\dot{\alpha}}, \hat{\bar{\pi}}^{p}{ }_{\dot{\alpha}}$ are eliminated. The Dirac brackets (and their Grassmannian generalizations 23) are decoupled by the introduction of $p_{+}{ }^{p}{ }_{a}$, 21,27 via

$$
\begin{aligned}
& \hat{\pi}^{p}{ }_{\alpha}=2 \pi^{p}{ }_{\alpha} \\
& \hat{p}^{p}{ }_{a}=p_{+}{ }^{p}{ }_{a}-\frac{V}{2} h^{1 / 2} \varepsilon^{p q r} \psi_{q}{ }^{\alpha} \sigma_{a \alpha \dot{\alpha}} \mathcal{C}_{r s}^{\alpha \beta} \pi_{\beta}^{s}
\end{aligned}
$$

with

$$
\mathcal{C}_{p q}^{\alpha \alpha}=-\frac{1}{2 V h^{1 / 2}}\left(i h_{p q} n^{a}-\varepsilon_{p q r} e^{r a}\right) \bar{\sigma}_{a}^{\dot{\alpha} \alpha}
$$

Here $\sigma_{a}$ are the $\sigma$-matrices in the conventions of [20], $V$ is the volume of the compact or compactified spacelike homogeneity surface $V=\int \boldsymbol{\omega}^{1} \wedge \boldsymbol{\omega}^{2} \wedge \boldsymbol{\omega}^{3}$, and $n^{a}$ is the future-oriented time-like unit vector orthogonal to the space-like homogeneity surfaces. It must be kept in mind that $n^{a}$ is a function of the tetrad components $e_{p}{ }^{b}$. The only non-vanishing Dirac brackets now are $\left\{e_{p}{ }^{a}, p_{+}{ }^{q}{ }_{b}\right\}^{*}=\delta_{p}{ }^{q} \delta_{b}{ }^{a}$ and $\left\{\pi_{p}{ }^{\alpha}, \psi^{q}{ }_{\beta}\right\}^{*}=-\delta_{p}{ }^{q} \delta_{\beta}{ }^{\alpha}$. The supersymmetry generators $S_{\alpha}, \bar{S}_{\dot{\alpha}}$ and Lorentz generators $J_{\alpha \beta}, \bar{J}_{\dot{\alpha} \dot{\beta}}$ in this representation are obtained as

$$
\begin{aligned}
& S_{\alpha}=-\left(\frac{1}{2} V m^{p q} e_{q}{ }^{a}+\frac{i}{2} p_{+}{ }^{p a}\right) \sigma_{a \alpha \dot{\alpha}} \mathcal{C}_{p r}^{\dot{\alpha} \beta} \pi_{\beta}^{r} \\
& \bar{S}_{\dot{\alpha}}=\left(\frac{1}{2} V m^{p q} e_{q}{ }^{a}-\frac{i}{2} p_{+}{ }^{p a}\right) \sigma_{a \alpha \dot{\alpha}} \psi_{p}{ }^{\alpha}
\end{aligned}
$$

and

$$
\begin{aligned}
J_{\alpha \beta}= & +\frac{1}{2}\left(\sigma^{a c} \epsilon\right)_{\alpha \beta}\left(e_{p a} p_{+}{ }^{p}{ }_{c}-e_{p c} p_{+}{ }^{p}{ }_{a}\right) \\
& -\frac{1}{2}\left(\psi_{p \alpha} \pi^{p}{ }_{\beta}+\psi_{p \beta} \pi^{p}{ }_{\alpha}\right) \\
\bar{J}_{\dot{\alpha} \dot{\beta}}= & -\frac{1}{2}\left(\epsilon \bar{\sigma}^{a c}\right)_{\dot{\alpha} \dot{\beta}}\left(e_{p a} p_{+}{ }^{p}{ }_{c}-e_{p c} p_{+}{ }^{p}{ }_{a}\right) .
\end{aligned}
$$

We have checked that the Dirac bracket $H_{\alpha \dot{\alpha}}=$ $-2 i\left\{S_{\alpha}, \bar{S}_{\dot{\alpha}}\right\}^{*}$ differs only by terms proportional to $J_{\alpha \beta}$ or $\bar{J}_{\dot{\alpha} \dot{\beta}}$ from the expression $\tilde{H}_{\alpha \dot{\alpha}}=\sigma_{a \alpha \dot{\alpha}}\left(e_{p}{ }^{a} \mathcal{H}^{p}+n^{a} \mathcal{H}\right)$ defined by the generators of the Hamiltonian $\mathcal{H}$ and diffeomorphism constraint $\mathcal{H}^{p}$ constructed in the canonical formulation.

Canonical quantization is achieved by the requirements $p_{+}{ }^{p}{ }_{a}=-i \hbar\left(\partial / \partial e_{p}{ }^{a}\right)$ and $\pi^{p}{ }_{\alpha}=-i \hbar\left(\partial / \partial \psi_{p}{ }^{\alpha}\right)$. In $S_{\alpha}$ there is then an ordering ambiguity between $p_{+}{ }^{p}{ }_{a}$ and $\mathcal{C}_{p r}^{\dot{\alpha} \beta}$, which we have resolved by adopting the ordering as written in eq. (3), see also 21. Using the quantized generators we then have computed their algebra. The result of these calculations, which are quite lengthy, in particular for establishing eqs. (7), (8), is as follows:

$$
\begin{aligned}
& {\left[S_{\alpha}, S_{\beta}\right]_{+}=0=\left[\bar{S}_{\dot{\alpha}}, \bar{S}_{\dot{\beta}}\right]_{+}} \\
& {\left[S_{\alpha}, \bar{S}_{\dot{\alpha}}\right]_{+}=-\frac{\hbar}{2} H_{\alpha \dot{\alpha}}}
\end{aligned}
$$

The operator $H_{\alpha \dot{\alpha}}$ is here defined by the anticommutator (6) in accordance with the classical Dirac bracket. The commutation relations of all operators with $J_{\alpha \beta}$ and $\bar{J}_{\dot{\alpha} \dot{\beta}}$ need not be written down explicitely, as they simply reflect the transformation properties of spinors and Lorentz-tensors in the spinor-formalism. In particular $S^{\alpha} S_{\alpha}$ and $\bar{S}_{\dot{\alpha}} \bar{S}^{\dot{\alpha}}$ are Lorentz scalars and therefore commute with $J_{\alpha \beta}, \overline{J_{\dot{\alpha}} \dot{\beta}}$.

$$
\begin{aligned}
{\left[H_{\alpha \dot{\alpha}}, \bar{S}_{\dot{\beta}}\right]_{-} } & =i \hbar \varepsilon_{\dot{\alpha} \dot{\beta}} D_{\alpha}^{\beta \gamma} J_{\beta \gamma} \\
{\left[H_{\alpha \dot{\alpha}}, S_{\beta}\right]_{-}=} & -i \hbar \varepsilon_{\alpha \beta} \bar{J}_{\dot{\beta} \dot{\gamma}} \bar{D}_{\dot{\alpha}}^{\dot{\beta} \dot{\gamma}} \\
= & -i \hbar \varepsilon_{\alpha \beta}\left[\bar{D}_{\dot{\alpha}}^{\dot{\beta} \dot{\gamma}} \bar{J}_{\dot{\beta} \dot{\gamma}}+i \hbar E_{\dot{\alpha}}^{\gamma \delta} J_{\gamma \delta}\right. \\
& \left.-i \hbar\left(1 / V h^{1 / 2}\right) n^{a} \varepsilon_{\dot{\alpha} \dot{\beta}} \bar{\sigma}_{a}^{\dot{\beta} \gamma} S_{\gamma}\right]
\end{aligned}
$$

The commutators $\left[\bar{S}_{\dot{\beta}}, H_{\alpha \dot{\alpha}}\right]_{-}$and $\left[S_{\beta}, H_{\alpha \dot{\alpha}}\right]_{-}$are the essential new results, on which all of the following is based. 
It should be noted, in particular, that only Lorentz generators appear on the right hand side of eq. (7). The operators $D_{\alpha}^{\beta \gamma}, \bar{D}_{\dot{\alpha}}^{\dot{\beta} \dot{\gamma}}, E_{\dot{\alpha}}^{\beta \gamma}$ are odd and functions of $\psi_{p}{ }^{\alpha}, \pi^{p}{ }_{\alpha}, e_{p}{ }^{a}, p_{+}{ }^{p}{ }_{a}$, whose explicit form we have determined, but will not be important here. The results (5)(8) demonstrate that the algebra of the generator closes, a fact which was always assumed in the earlier work on the same minisuperspace models, but which is here established explicitely by eqs. (7), (8).

Let us now find the physical states of these models, given by all the states which are annihilated by the generators $S_{\alpha}, \bar{S}_{\dot{\alpha}}, J_{\alpha \beta}, \bar{J}_{\dot{\alpha} \dot{\beta}}, H_{\alpha \dot{\alpha}}$. The Lorentz generators automatically annihilate all states which are Lorentz scalars. Therefore, it is sufficient to demand that physical states are Lorentz scalars and annihilated by $S_{\alpha}$ and $\bar{S}_{\dot{\alpha}}$; their annihilation by $H_{\alpha \dot{\alpha}}$ is then automatically guaranteed due to eq. (6). The form of the constraint operators guarantees that physical states have a fixed fermion number $F=\psi_{p}{ }^{\alpha} \partial / \partial \psi_{p}^{\alpha}$, given by the number of factors of $\psi_{p}{ }^{\alpha}$ in the $\psi$-representation: $F$ must be an even number in Lorentz-invariant states and ranges from 0 to 6 in the present models.

The physical states in the sectors $F=0$ and $F=6$ are easily obtained, and are, respectively, given by

$$
\begin{aligned}
& \Psi_{0}=\text { const } e^{\frac{V}{2 \hbar} m^{p q} h_{p q}} \\
& \Psi_{6}=\text { const } h e^{-\frac{V}{2 \hbar} m^{p q} h_{p q}} \prod_{r}\left(\psi_{r}\right)^{2}
\end{aligned}
$$

reproducing a well known result [7]- [12].

In order to show that there exist physical states in the 2 -fermion sector let us consider the wave-function

$$
\Psi_{2}=\bar{S}_{\dot{\alpha}} \bar{S}^{\dot{\alpha}} f\left(h_{p q}\right),
$$

where we require, of course, that $\bar{S}_{\dot{\alpha}} \bar{S}^{\dot{\alpha}} f \neq 0$. Here $f$ is a function of the $h_{p q}$ only, and therefore, like $\bar{S}_{\dot{\alpha}} \bar{S}^{\dot{\alpha}}$, a Lorentz scalar. Therefore $\Psi_{2}$ automatically satisfies the Lorentz constraints and the $\bar{S}$-constraints because of eq. (5). The only remaining condition is $S_{\alpha} \Psi_{2}=0$, which, after the use of eqs. (10), (6), reduces to

$$
\left[H_{\alpha \dot{\alpha}}, \bar{S}^{\dot{\alpha}}\right]_{-} f \quad+2 \bar{S}^{\dot{\alpha}} H_{\alpha \dot{\alpha}} f \quad=0
$$

The first term is proportional to $J_{\beta \gamma}$ thanks to eq. (7) and therefore vanishes because $f$ is a Lorentz scalar. The second term vanishes if $f$ satisfies the Wheeler-DeWitt equation 24

$$
H_{\alpha \dot{\alpha}}{ }^{(0)} f\left(h_{p q}\right)=0
$$

where $H_{\alpha \dot{\alpha}}{ }^{(0)}$ consists only of the bosonic terms of $H_{\alpha \dot{\alpha}}$, i.e. of the terms which remain if $\pi^{p}{ }_{\alpha}$ is brought to the right and then equated to zero. Any solution of this Wheeler-DeWitt equation, which may be specified further, e.g. by imposing Hartle-Hawking [2] no-boundary conditions, or Vilenkin [2] tunneling boundary conditions, or wormhole boundary conditions [25], or some choice of scalar product [3], generates a solution in the 2-fermion sector via eq. (10), with a definite dependence on the fermionic variables, which are only present in the $\bar{S}_{\dot{\alpha}} \bar{S}^{\dot{\alpha}}$-term.

Let us observe now that the norm of $\Psi_{2}$ vanishes due to the appearance of $\bar{S}_{\dot{\alpha}}$ in eq. (10) and the fact that $S_{\alpha}$ is the adjoint of $\bar{S}_{\dot{\alpha}}$. However, a proper definition of the scalar product, which we shall not attempt here, must also include some gauge-fixing condition in its measure. The norm of $\Psi_{2}$ (and of $\Psi_{4}$ to be considered below) in such a properly defined scalar product will then not vanish.

Let us now turn to physical states in the 4 -fermion sector. Similarly to eq. (10) the wave-function

$$
\Psi_{4}=S^{\alpha} S_{\alpha} g\left(h_{p q}\right) \prod_{r=1}^{3}\left(\psi_{r}\right)^{2} .
$$

automatically satisfies the Lorentz constraints and the $S$ constraint. It remains to satisfy the $\bar{S}_{\dot{\alpha}}$ constraint, which reduces to

$$
\left(\left[H_{\alpha \dot{\alpha}}, S^{\alpha}\right]_{-}+2 S^{\alpha} H_{\alpha \dot{\alpha}}\right) g\left(h_{p q}\right) \prod_{r=1}^{3}\left(\psi_{r}\right)^{2}=0 .
$$

Let us consider the first term in the bracket: By the use of equation (8) it is expanded in terms containing the Lorentz generators or $S_{\gamma}$ as factors on the right. The terms containing the Lorentz generators vanish as they act on Lorentz scalars. In the term containing $S_{\gamma}$, the generator $S_{\gamma}$ can be brought to the left because it happens to commute with its prefactor. Combining this term with the second term in the bracket of eq. (14), $S^{\alpha}$ can be factored out to the left. To fulfill eq. (14) it is therefore enough [24] if $g$ satisfies the Wheeler-DeWitt equation.

$$
\left(H_{\alpha \dot{\alpha}}{ }^{(1)}-\frac{\hbar^{2}}{V h^{1 / 2}} n^{a} \sigma_{a \alpha \dot{\alpha}}\right) g\left(h_{p q}\right)=0
$$

where $H_{\alpha \dot{\alpha}}{ }^{(1)}$ consists of those terms of $H_{\alpha \dot{\alpha}}$ which remain if the $\pi^{p}{ }_{a}$ are brought to the left and then equated to zero. The resulting Wheeler-DeWitt equation is slightly different from that obeyed by the amplitude in the 2 -fermion sector, but apart from this the degree of generality of the solution is the same.

Our results differ from earlier work on the supersymmetric Bianchi models in class A [8]- [12] which concluded that physical states in the 2- and 4-fermion sectors do not exist. In hindsight, this conclusion can be traced to an overly restricted ansatz for Lorentz-invariant wavefunctions [26]: Lorentz invariants were constructed only from the irreducible spin- $1 / 2$ and spin- $3 / 2$ components contained in the Rarita-Schwinger field $\psi_{p}{ }^{\alpha}$ (there are only 2 such invariants bilinear and two more quadri-linear 
in $\psi_{p}{ }^{\alpha}$ ), omitting the further invariants which can be formed with help of the irreducible spin- 2 components contained in the gravitational degrees of freedom. In fact, such components can be used to generate up to $\left(\begin{array}{l}6 \\ 2\end{array}\right)=15$ Lorentz invariants in the 2 -fermion sector. A simple example of such an additional invariant is $m^{p q} \psi_{p}{ }^{\alpha} \psi_{q \alpha}$ another one is $m^{p r} h_{r s} m^{s q} \psi_{p}{ }^{\alpha} \psi_{q \alpha}$ etc. Writing out the expressions (10), (12) for $\Psi_{2}$ and $\Psi_{4}$ in an explicit way it can be seen that indeed they contain such additional invariants.

We finish by speculating on a possible generalization of these solutions for the case of full supergravity. Provided the algebra of the local generators of the constraints still has a form like eqs. (6)-(8) physical states still exist which look somewhat like $\Psi_{2}$ and $\Psi_{4}$, but contain formal products of $(\bar{S})^{2}$ or $(S)^{2}$ over all points of the space-like 3surface, thus leading to states with infinite fermion number. Such states have recently been discussed in 17]. The new physical states we have found in the present paper are the direct minisuperspace analogues of such states in full supergravity; even though, due to the reduction to minisuperspace the fermion number is, of course, finite (in fact just 2 or 4 ). It is remarkable that the new states display the same richness of gravitational dynamical behavior as the Bianchi models in pure gravity. In this respect they differ qualitatively from the earlier found states in the empty and the full fermion sectors, which are highly symmetric and do not describe the full dynamical behavior of the Bianchi models in the classical limit, which, as is well known, can be very asymmetric and rich.

While the states in the empty and filled sectors alone would span at most a 2-dimensional Hilbert space, the new physical states described here span an infinitedimensional Hilbert space just as in the Bianchi models of pure gravity [3]. Just how this Hilbert space ought to be constructed is, of course, one of the questions one hopes to unravel by the further study of minisuperspace models. That this strategy is not only fruitful in the case of pure gravity but can also be usefully employed in the case of supergravity is an encouraging conclusion of the present paper.

This work has been supported by the Deutsche Forschungsgemeinschaft through the Sonderforschungsbereich 237 "Unordnung und große Fluktuationen". One of us (A. Csordás) would like to acknowledge additional support by The Hungarian National Scientific Research Foundation under Grant number F4472.

[1] J.A. Wheeler, in "Relativity Groups and Topology", ed. C. DeWitt and B. DeWitt, (Gordon and Breach, New
York 1964); B.S. DeWitt, Phys. Rev. 1601113 (1967); C. W. Misner, in "Magic without Magic", ed. J. Klauder (Freeman, San Francisco 1972).

[2] J. B. Hartle and S. W. Hawking, Phys. Rev. D28, 2960 (1983); A. Vilenkin, Phys. Rev. D33, 35-60 (1986)

[3] A. Ashtekar, R. S. Tate and C. Uggla, Int. J. Mod. Phys. D2, 15 (1993); D. Marolf (preprint gr-qc/9409049)

[4] A. Macías, O. Obregón, and M. P. Ryan Jr., Class. Quantum Grav. 4, 1477 (1987)

[5] P. D. D'Eath and D. I. Hughes, Phys. Lett. B214, 498 (1988); Nucl. Phys. B378, 381 (1991)

[6] J. Socorro, O. Obregón, and A. Macías, Phys. Rev. D45, 2026 (1992); O. Obregón, J. Socorro and J. Benitez, ibid 47, 4471 (1993)

[7] R. Graham Phys. Rev. Lett 67, 1381 (1991); Phys. Lett. B277, 393 (1992); J. Bene and R. Graham, Phys. Rev. D49, 799 (1994)

[8] P. D. D'Eath, S. W. Hawking and O. Obregón, Phys. Lett. B300, 44 (1993)

[9] P. D. D'Eath, Phys. Rev. D48, 713 (1993)

[10] R. Graham Phys. Rev. D48, 1602 (1993)

[11] M. Asano, M. Tanimoto, and N. Yoshino, Phys. Lett. B314, 303 (1993)

[12] R. Capovilla and J. Guven (preprint 1994, grqc/9402025

[13] P. D. D'Eath, Phys. Lett. B320, 12 (1994)

[14] A. D. Y. Cheng, P. D. D'Eath and P. R. L. V. Moniz, (preprint 1994, gr-qc/9404008); (preprint 1994, grqc/9406047)

[15] R. Capovilla, and O. Obregón (preprint 1994, grqc/9402042

[16] R. Graham and H. Luckock, Phys. Rev. D49, R4981 (1994)

[17] S. M. Carroll, D. Z. Freedman, M E. Ortiz, and D. N. Page, Nucl. Phys. B423, 661 (1994)

[18] P. D. D'Eath, Phys. Lett. B321, 368 (1994)

[19] G. F. R. Ellis and M. A. H. McCallum, Comm. Math. Phys. 12, 108 (1960)

[20] J. Wess and J. Bagger, "Supersymmetry and Supergravity", (Princeton University Press, Princeton 1972)

[21] P. D. D'Eath, Phys. Rev. D29, 2199 (1984)

[22] M. P. Ryan Jr. and L. C. Shepley, "Homogeneous Relativistic Cosmologies" (Princeton University Press, Princeton 1975)

[23] R. Casalbuoni, Nuovo Cimento 33A, 115 (1976)

[24] Whether the even more general possibility $H_{\alpha \dot{\alpha}}{ }^{(0)} f\left(h_{p q}\right)=C\left(n^{a} / h^{1 / 2}\right) \sigma_{a \alpha \dot{\alpha}} \exp \left(V m^{p q} h_{p q} / 2 \hbar\right)$ to satisfy (11), with arbitrary constant $C \neq 0$ (and a similar inhomogenous generalization of eq. (15), with $c n^{a} h^{1 / 2} \sigma_{a \alpha \dot{\alpha}} \exp \left(-V m^{p q} h_{p q} / 2 \hbar\right)$ appearing in the inhomogeneity) are relevant, too, can only be decided when the full Hilbert space of physical states is constructed. Here we take $C=0$.

[25] S. W. Hawking, Phys. Rev. D37, 904 (1988)

[26] In Ref. [10] solutions of the form (10) were actually looked for, but were there found not to exist, because in 10] the generators and their algebra were not derived from supergravity, and different from eqs. (5)-(8).

[27] H. Luckock and C. Oliwa, (preprint 1994, gr-qc/9412028) 